\title{
Growth of chronic myeloid leukemia cells is inhibited by infection with Ad-SH2-HA adenovirus that disrupts Grb2-Bcr-Abl complexes
}

\author{
ZHI PENG ${ }^{1 *}$, HONG-WEI LUO ${ }^{1 *}$, YING YUAN ${ }^{2}$, JING SHI $^{1}$, SHI-FENG HUANG ${ }^{3}$, \\ CHUN-LI LI ${ }^{1}$, WEI-XI CAO ${ }^{1}$, ZONG-GAN HUANG ${ }^{4}$ and WEN-LI FENG ${ }^{1}$
}

\begin{abstract}
${ }^{1}$ Department of Clinical Hematology, Key Laboratory of Laboratory Medical Diagnostics Designated by the Ministry of Education, Chongqing Medical University; Departments of ${ }^{2}$ General Surgery, ${ }^{3}$ Clinical Laboratory and

${ }^{4}$ Hematology, the First Affiliated Hospital of Chongqing Medical University, Chongqing 400016, P.R. China
\end{abstract}

Received December 13, 2010; Accepted February 2, 2011

DOI: 10.3892/or.2011.1197

\begin{abstract}
The persistence of Bcr-Abl-positive cells in patients on imatinib therapy indicates that inhibition of the Bcr-Abl kinase activity alone might not be sufficient to eradicate the leukemia cells. Many downstream effectors of Bcr-Abl have been described, including activation of both the Grb2SoS-Ras-MAPK and Grb2-Gab2-PI3K-Akt pathways. The Bcr-Abl-Grb2 interaction, which is mediated by the direct interaction of the Grb2 SH2 domain with the phospho-Bcr-Abl $\mathrm{Y} 177$, is required for activation of these signaling pathways. Therefore, disrupting their interaction represents a potential therapeutic strategy for inhibiting the oncogenic downstream signals of Bcr-Abl. Adenovirus Ad-SH2-HA expressing the Grb2 SH2 domain was constructed and applied in this study. As expected, Ad-SH2-HA efficiently infected CML cells and functioned by binding to the phospho-Bcr-Abl Y177 site, competitively disrupting the Grb2 SH2-phospho-Bcr-Abl Y177 complex. They induced potent anti-proliferation and apoptosis-inducing effects in CML cell lines. Moreover, the Ras, MAPK and Akt activities were significantly reduced in the Ad-SH2-HA treated cells. These were not observed with the point-mutated control adenovirus Ad-Sm-HA with abolished phospho-Bcr-Abl Y177 binding sites. These data
\end{abstract}

Correspondence to: Professor Wen-Li Feng, Department of Clinical Hematology, Key Laboratory of Laboratory Medical Diagnostics Designated by the Ministry of Education, Chongqing Medical University, Chongqing 400016, P.R. China

E-mail: fengwlcqmu@sina.com

Abbreviations: CML, chronic myeloid leukemia; Grb2, growth factor receptor-bound protein 2; CAR, coxsackie adenovirus receptor; MOI, multiplicities of infection; FCS, fetal calf serum; MTT, thetetrazolium dye,3-(4,5-dimethylthiazol-2-yl)-2,5-diphenyltetrazolium bromide; HRP, horseradish peroxidase

Key words: Bcr-Abl, $\mathrm{SH} 2$ domain, growth factor receptor-bound protein 2, Ras, MAPK, Akt indicate that, in addition to the direct targeting of Bcr-Abl, selective inhibition of its downstream signaling pathways may be a therapeutic option for CML, and the Ad-SH2-HAmediated killing strategy could be explored as a promising anti-leukemia agent in CML.

\section{Introduction}

The Bcr-Abl oncoprotein is responsible for most cases of chronic myeloid leukemia (CML), with the deregulated ABL tyrosine kinase of Bcr-Abl fusion protein playing a major causative role in its pathogenesis (1). State-of-the-art therapies for CML patients currently include cytostatic drugs such as $\alpha$-interferon, small tyrosine kinase inhibitors such as imatinib, dasatanib and nilotinib, as well as bone marrow or stem cell transplantation for younger patients (2).

Although imatinib is a remarkably effective treatment for chronic phase CML, imatinib resistance does occur, particularly in blast crisis patients and usually as a result of mutations in Bcr-Abl, in particular the prevalent T315I mutation (3). This has led to efforts to develop second generation BCR-ABL inhibitors that can block the activity of these imatinib-resistant mutant forms of Bcr-Abl. In clinical trials, dasatanib, a dual c-Src and c-Abl inhibitor, proved effective in CML therapy and works against most of the imatinib-resistant Bcr-Abl mutations but not against T315I. Nilotinib, which is also active against most of the imatinib-resistant mutations but T315I Bcr-Abl, has also shown promising results in clinical trials for CML and is expected to be approved soon (4). To expedite the identification of strategies to override the resistance imposed by the T315I mutation, several strategies have been pursued, including the exploitation of BCR-ABL1 kinase sites distant from the ATP-binding pocket to cripple the kinase activity of the enzyme and inhibiting signaling pathways downstream from BCR-ABL1. Recent insights gained regarding the structural biology of T315I have led to the development of a variety of compounds against this mutant (5). However, the current treatment options are not yet satisfactory with respect to drug resistance and the poor long-term survival of the patients. It is therefore important to propose alternative therapeutics. 
Although Bcr-Abl remains an attractive therapeutic target, it is important to identify other components involved in CML pathogenesis, especially those beyond the kinase activity of $\mathrm{BCR}-\mathrm{ABL}$, to overcome drug resistance.

The fusion of Bcr sequences to Abl during the translocation associated with CML not only increases the tyrosine kinase activity of ABL, but also brings new regulatory domains/ motifs to Abl, such as the growth factor receptor-bound protein 2 (Grb2) SH2-binding site, phospho-Bcr-Abl Y177. The autophosphorylated Bcr-Abl Y177 recruits Grb2 via its SH2 domain, which is required for efficient induction of the myeloproliferative disease by Bcr-Abl in a mouse BM retroviral transduction/transplantation model (6). Moreover, numerous signaling pathways, including the Grb2-SoS-RasMAPK and Grb2-Gab2-PI3K-Akt pathway, are activated upon the Grb2-Bcr-Abl interaction in CML cells $(7,8)$.

Given that the Bcr-Abl-Grb2 interaction, which is mediated by the direct interaction of the Grb2 SH2 domain with the phospho-Bcr-Abl Y177, is required for activation of the above mentioned oncogenic signaling pathways, disrupting their interaction through large excess of exogenous SH2 may represent a potential therapeutic strategy to specifically disrupt the oncogenic potential of Bcr-Abl.

Any effective gene therapy will firstly require a safe and highly efficient vehicle to carry the therapeutic gene into its target cells. Various virus vectors such as retroviruses and lenti-viruses have been widely used for genetic manipulation of malignant cells, however, due to the potential integration of the vector genes with the host chromatin material, safety remains a serious concern for the ultimate clinical application of various gene-expressing vectors (9-12). Adenoviral vectors have been widely used for transient genetic manipulation of malignant cells because the vector genome normally does not integrate into the host cell chromosomes and will ultimately be lost or degraded upon multiple cell divisions, thus providing a safe gene transfer mode. Moreover, they harbor active mechanisms for entry into the host cells and the intracellular trafficking of the internalized vector genome into the nucleus, thus transient, but high level gene expression is expected upon adenoviral vector mediated gene delivery. In addition, adenoviral vectors can infect both dividing and non-dividing cells $(13,14)$. Nonetheless, due to a paucity of CAR, the transfer of the adenovirus serotype 5 (Ad5)-based vector-mediated gene into malignant hematopoietic cells was greatly hampered. In contrast, fiber-retargeted adenoviral vectors with species $\mathrm{B}$ tropism can potentially bypass the CAR requirement and facilitate efficient gene transfer into malignant hematopoietic cells (15). Thus the Ad5f35 system, which has an Ad5 backbone with a chimeric Ad5/Ad35 fiber, was selected in the present study for its ability to infect hematopoietic cells.

In the present study, The Ad5f35 system was employed to deliver the exogenous $\mathrm{SH} 2$ domain of the Grb2 protein into K562 and KU812 CML cells. SH2 and HA fragments were subsequently cloned into Ad5f35 vector, in which the HA tag was designed to allow easy demonstration of co-immunoprecipitation between the exogenous $\mathrm{SH} 2$ peptide and the Bcr-Abl oncoprotein. The Ad-SH2-HA adenovirus expressing the Grb2 $\mathrm{SH} 2$ domain that was expected to competitively disrupt the Grb2 SH2-phospho-Bcr-Abl Y177 complex was constructed and tested. Ad-SH2-HA was analyzed for its ability to infect
CML cells, to efficiently express exogenous $\mathrm{SH} 2$ peptide, to heterodimerize with $\mathrm{Bcr}-\mathrm{Abl}$, to inhibit the growth of $\mathrm{CML}$ cells, and to further explore the kinase-inhibiting effect of the Ad-SH2-HA virus on Bcr-Abl downstream effectors such as Ras, MAPK and Akt. As expected, Ad-SH2-HA efficiently infected into CML cells and functioned by potently binding to the phospho-Bcr-Abl Y177 site. They induced a dramatic decrease in the growth of CML cell lines, which was not observed with the point-mutated control adenovirus Ad-Sm-HA with abolished phospho-Bcr-Abl Y177 binding site. Moreover, reduction of Ras, MAPK and Akt activities was observed in the Ad-SH2-HA-treated cells. These results encourage future tests of inhibitors developed to block Grb2-Bcr-Abl protein interactions in the context of Bcr-Abldependent diseases.

\section{Materials and methods}

Construction of the Ad5/f35 adenoviral vectors and adenovirus transduction. The 279 bp SH2 (Homo sapiens, GenBank accession no. NP_002077) cDNA was cloned from a human placenta cDNA library by polymerase chain reaction (PCR) using the following primers: F, 5'-TGGTTTTTTGGCAAAATC-3' and R, 5'-AAGGACGCCCTGTATCTT-3'. The SH2 domain of Grb2 was selected for its high binding affinity to Bcr-Abl Y177. To show the specific binding between the SH2 domain of Grb2 and the phospho-Bcr-Abl Y177, we additionally designed a mutant sequence $(\mathrm{Sm})$ in which two base pairs were mutated, leading to one amino acid mutation (R27K) in the binding domain, which was expected to hinder its binding to phospho-Bcr-Abl Y177 (Fig. 1). The SH2 (R27K) mutant was created by oligonucleotide site-directed mutagenesis using the Muta-Gene Phagemid in vitro mutagenesis system (Stratagene) as described (16).

The Ad5/f35GFP vector was supplied by Dr Xiaolong Fan (Lund University, Sweden). Preparation of cDNAs for HA-tag, $\mathrm{SH} 2$ and Sm into pAdTrack-CMV vectors were performed as previously described (17). This shuttle plasmid was used to generate a recombinant adenoviral genome encoding Ad5F35-GFP by homologous recombination in Escherichia coli BJ5183 with the fiber gene modified pAdEasy-1/F35 by using a standard method. The genomic plasmid encoding Ad5F35-GFP was digested with PacI and transfected into 293 cells. The adenoviral vectors were expanded in 293 cells, purified and titered in 293 cells by serial dilutions according to methods described previously $(17,18)$. All multiplicities of infection (MOIs) used in this study are based on virus particles (vp); 1 infectious unit (iu) $=10^{5} \mathrm{vp}$. For gene transduction, cells were incubated with adenoviral aliquots for $4 \mathrm{~h}$ before addition of culture medium. Ad5/F35-Null virus was used as a non-specific control for gene transfer. The transduction efficiency was evaluated by observation of GFP expression under fluorescent microscope at $48 \mathrm{~h}$ after transduction.

Cell culture. The human Bcr-Abl (p210)-positive CML cells (K562, KU812) and HL60 leukemic promyelocytic cells were maintained in RPMI-1640 medium (Gibco) with 10\% FCS. ECV304 cells were maintained in Dulbecco's modified Eagle's medium (Gibco) with 10\% FCS. All cells were cultured in a humidified incubator at $37^{\circ} \mathrm{C}$ and $5 \% \mathrm{CO}_{2}$. 
MTT assay. K562, KU812, HL60 and ECV304 cells $\left(0.5 \times 10^{6} / \mathrm{ml}\right)$ infected with viruses were washed three times with PBS, The cells were suspended with medium (10\% FCS), and plated in a 96-microwell plate at a concentration of $1 \times 10^{5}$ cells/per well. The MTT assay was performed as we have previously reported (19). Briefly, three replicate wells were used for each treated group. At end of this time, $20 \mu 1$ MTT solution $(5 \mathrm{mg} / \mathrm{ml})$ was added to each well and plates were returned to incubator for $4 \mathrm{~h}$ at $37^{\circ} \mathrm{C}$. To pack cells to the base of wells, plates were centrifuged at $500 \mathrm{~g}$ for $5 \mathrm{~min}$ and the bulk of the medium was removed. DMSO (100 $\mu$ l) was then added to each well to disrupt the cells and dissolve the crystalline formazan deposits. Plates were agitated gently on a plate shaker for $10 \mathrm{~min}$, and the absorbance was read at $570 \mathrm{~nm}$.

Immunocytochemistry and confocal microscopy. K562 cells were infected by viruses, and prepared in $48 \mathrm{~h}$ post-transduction. Briefly, cells were fixed in $100 \%$ pre-chilled methanol at $-20^{\circ} \mathrm{C}$ for $20 \mathrm{~min}$. Following three washes with PBS, slides were permeabilized with $0.3 \%$ Triton X-100/PBS for $15 \mathrm{~min}$ at $37^{\circ} \mathrm{C}$, blocked in $10 \%$ normal goat serum for $1 \mathrm{~h}$ at $37^{\circ} \mathrm{C}$ and incubated with mouse anti-human HA-tag primary antibody (Cell Signal, MA, USA) at 1:25 in 10\% normal goat serum/PBS at $4^{\circ} \mathrm{C}$ overnight followed by three washes with PBS (pH 7.2) containing 0.5\% Triton X-100 (Sigma, St. Louis, $\mathrm{MO})$. The cells were further incubated with goat anti-mouse Cy3-IgG (Molecular Probes, Eugene, OR), washed and stained with DAPI for $10 \mathrm{~min}$. After three washes with PBS, the cells were treated with a fluorescent mounting medium (Dako) and visualized by confocal microscopy (Leica Lasertech $\mathrm{GmbH}$, Heidelverg, Germany). Fluorescence images were acquired by means of a PL-APO 100X objective. In order to confirm the cellular localization in the 3-D images, 4-sectioned images were overlapped into a single image at the same phase as we previously reported $(20,21)$.

Flow cytometric analysis for leukemia cell apoptosis. For all flow cytometric analyses, a FACS Calibur instrument [BectonDickinson (BD), Mountain View, CA] was used to acquire, and CellQuest software (BD) to analyze data. Apoptosis was assessed on day 5 post-infection by flow cytometry after labeling with Annexin V-PE and 7-AAD (BD PharMingen). Cells were washed once with washing buffer, pelleted and antibodies were added to the pellet in saturating amounts $(5 \mu \mathrm{l})$. After 15 -min incubation at $4^{\circ} \mathrm{C}$ in the dark, cells were washed thrice, fixed and analyzed.

Western blotting. M-PER Mammalian Protein Extraction Reagent supplemented with proteinase and phosphatase inhibitors (cell signaling) was employed to lyze cells. Protein were resolved on $8-12 \%$ SDS-PAGE gels and transferred to PVDF membranes. Membranes were blocked with 5\% BSA in TBS and $0.1 \%$ Tween-20, and respectively labeled with primary antibodies anti-HA (Cell Signal), anti- $\beta$-actin (Santa Cruz Biotechnology), p-Bcr (Tyr 177) (Santa Cruz Biotechnology), anti-Grb2 (Santa Cruz Biotechnology), anti-Akt and antip-Akt (Cell Signal), anti-p-Erk (Santa Cruz Biotechnology), anti-MAPK and anti-p-MAPK (Santa Cruz Biotechnology), followed by HRP-conjugated anti-mouse or anti-rabbit antibodies (1:5000; Santa Cruz Biotechnology). Membranes were sequentially reprobed with antibodies to phosphospecific and total proteins and to $\beta$-actin, and blots were developed using Supersignal WestPico chemiluminescent substrate (Pierce), imaged and analyzed by the Bio-Rad Gel Imaging System as previously reported (22).

Co-immunoprecipitation. M-PER Mammalian Protein Extraction Reagent was employed to lyze the cells and the whole cell lysates from K562 cells treated with the Ad-SH2-HA, Ad-Sm-HA, Ad5F35-GFP or PBS were immunoprecipitated with anti-HA agarose resin according to the manual of the ProFound ${ }^{\text {Ti }}$ Mammalian HA-Tag IP/Co-IP Kit (Pierce). The precipitate-bound beads were eluted with $2 \mathrm{X}$ non-reducing sample buffer. Eluates from the immunoprecipitation beads from cell extracts were separated by a 6-12\% gradient SDS-PAGE gel and then electrophoretically transferred to PVDF membrane (Millipore, Bedford, MA) following the manufacturer's protocol. Immunoblotting was then performed using anti-p-Bcr177 or anti-HA (Cell Signal) as the primary antibodies. Goat anti-rabbit or goat antimouse $\operatorname{IgG}$ conjugated to HRP was used respectively as the secondary antibody. Blots were developed using Supersignal WestPico chemiluminescent substrate (Pierce), imaged and analyzed by the Bio-Rad Gel Imaging System.

For the second step, the protein extract was precleaned with protein A-Sepharose beads (Pierce Chemical Co.) at $4^{\circ} \mathrm{C}$ for $1 \mathrm{~h}$. Primary anti-p-Bcr177 antibody was added to protein extracts and incubated overnight at $4^{\circ} \mathrm{C}$, followed by incubation with $30 \mu \mathrm{l}$ True Blot beads (eBioscience) for $2 \mathrm{~h}$. Beads were isolated by centrifugation, washed with PBS plus $1 \%$ NP40, and boiled with $2 \mathrm{X}$ sample loading buffer, and the immunoprecipitated proteins were separated by SDS-PAGE and subjected to Western blotting with anti-HA and anti-pBcr177 as described above (23).

Ras, MAPK and Akt kinase assays. The in vitro kinase assays for Ras, MAPK and Akt were performed as previously reported (23). Briefly, Ras activation assay kit (Pierce) was used per the manufacturer's instructions. Cell lysates were prepared, and aliquots were set aside to allow quantitation of total Ras. The remaining lysate was mixed with $10 \mu \mathrm{l}$ $0.5 \mathrm{mmol} / \mathrm{l}$ EDTA (pH 8.0) and $5 \mu 1$ of $10 \mathrm{mmol} / 1 \mathrm{GTP} \gamma \mathrm{S}$ and incubated at $30^{\circ} \mathrm{C}$ for $15 \mathrm{~min}$. The mixture was added to a column containing immobilized GST-Raf1-RBD fusion protein and incubated on ice for $1 \mathrm{~h}$. Bound protein was eluted with $2 X$ SDS sample buffer. Glutathione S-transferase (GST)bound Ras (active Ras) and total Ras were detected by Western blotting with an anti-Ras antibody. Akt kinase or p44/42 MAP kinase (MAPK) assay kits (Cell Signaling Technology) were used following the manufacturer's instructions. Akt or p44/42 MAPK proteins were immunoprecipitated, and in vitro kinase reactions were done using glycogen synthesis kinase-3 (GSK-3) and ErK-1 fusion proteins as substrates, respectively. Reaction products were subjected to Western blotting with antibodies to phospho-GSK-3 $\alpha / \beta$ or phospho-ErK-1. One third of the lysate was retained for Western blotting for $\beta$-actin to check loading.

Statistical analysis. All comparisons were two-tailed, and p-values of $<0.05$ were considered significant. 
A

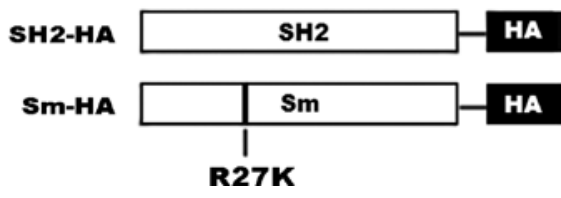

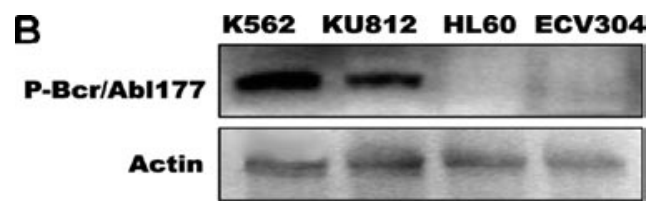
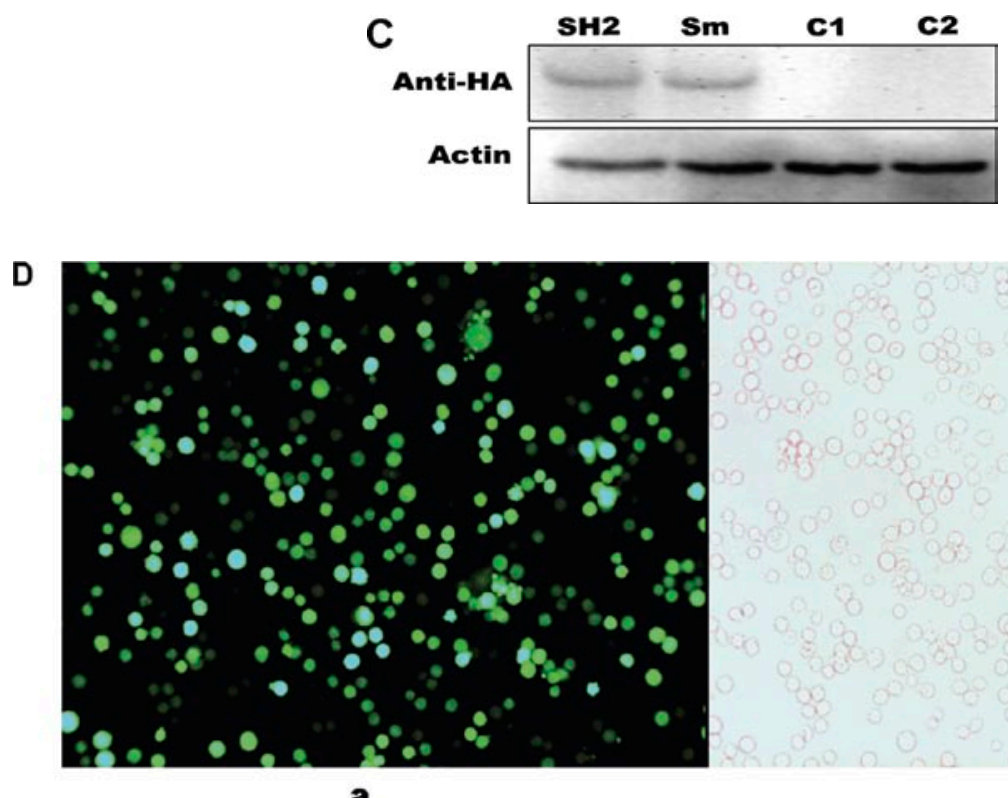

a

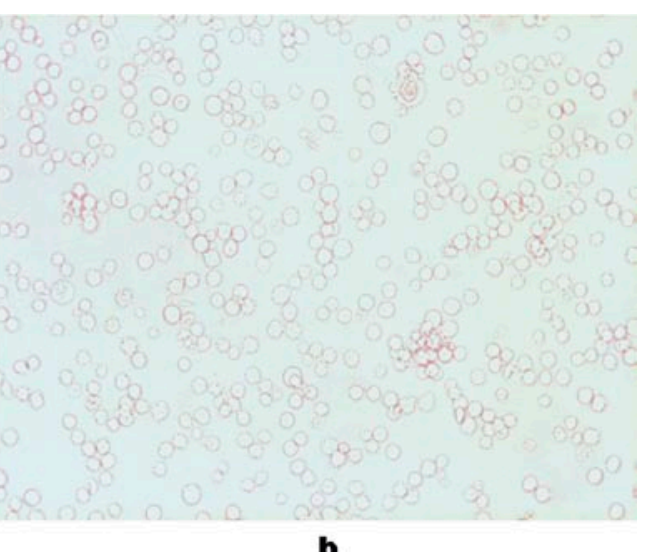

Figure 1. Construction of adenoviral vectors and confirmation of its infection efficiency. (A) Construction of the Ad-SH2-HA and Ad-Sm-HA vectors; (B) Western blotting was employed to confirm the expression of the exogenous SH2 in adenovirus-infected K562 cells using anti-HA antibody; (C) Western blotting was employed to detect the expression of phosphorylated Bcr-Abl tyrosine 177 (phospho-Bcr-Abl Y177) in both K562 and KU812 CML cell lines as described in Materials and methods; (D) Adenoviral GFP expression in K562 cells as observed under the inverted fluorescent microscope. K562 cells were infected with the Ad5/F35-GFP control adenovirus for $48 \mathrm{~h}$, and GFP expression was observed under the inverted fluorescent microscope (a) to determine virus titer. The image under light microscope (b) was also included.

\section{Results}

SH2-encoding adenoviral vector was successfully constructed, and adenovirus with high titer was generated. As shown in Fig. 1A, the control adenoviral vector pAd5F35GFP, the wild and mutant SH2-expressing pAd-SH2-HA and pAd-SH2-Sm were successfully constructed as proved by restriction enzyme digestions and sequencing (data not shown). The corresponding adenoviral stocks Ad5F35-GFP, Ad-SH2-HA and Ad-SH2-Sm with high virus titers are shown in Fig. 1D.

In vitro infection of the CML leukemia cells with adenoviral Ad5F35-GFP and Ad-SH2-HA. We first assessed the infection efficiency of Ad5F35-GFP virus in K562 CML cells. Efficient infection was confirmed by GFP expression analysis using fluorescence microscopy (Fig. 1D), whereas the expression of both the wild-type and the mutant exogenous $\mathrm{SH} 2$ in K562 cells was confirmed by Western blotting (Fig. 1B) and immunofluorescence (Fig. 2). Moreover, as is shown in Fig. 2B and C, expression of both the wild and mutant $\mathrm{SH} 2$ was detected exclusively in the cytoplasm.

Ad-SH2-HA infection inhibits CML cell proliferation. To test the hypothesis that Ad-SH2-HA infection would influence the growth of CML cells, we evaluated the effect of Ad-SH2-HA infection on cell growth using MTT assay. When compared with those of the PBS-treated or Ad5/F35-GFP-infected cells (Fig. 3A), while pre-infection with Ad-Sm-HA hematological only induced minor reduction in cell proliferation, the K562 cells pre-infected with Ad-SH2-HA showed dramatic growth inhibition in a time-dependent manner. When cells of different origins, including Bcr-Abl positive K562 and KU812 CML cells, Bcr-Abl negative HL-60 cells of the hematological origin and the Bcr-Abl negative ECV304 cells of the endothelial cell origin were tested, we respectively observed a $39 \%$ and $35 \%$ inhibition of cell growth for K562 and KU812 cells at an MOI of 20000, which were significantly higher than those for both HL-60 and ECV304 cells (Fig. 3B). While the K562 cells demonstrated significantly higher basal phospho-Bcr-Abl Y177 protein expression level than that of the KU812 cells (Fig. 1C), K562 showed more potent sensitivity to Ad-SH2-HA infection at the same MOI, suggesting that K562 is more sensitive to Ad-SH2-HA infection (Fig. 3B), whereas proliferation of K562 cells which were pre-infected by the mutant Ad-Sm-HA was unaffected despite equally efficient infection (Fig. 2B and C).

Ad-SH2-HA infection induced cell apoptosis in human CML cells. A large percentage of the two CML cell lines, K562 and KU812, were in the apoptotic fraction $96 \mathrm{~h}$ after Ad-SH2-HA infection, $30 \%$ and $28 \%$, compared with cells infected with Ad5/ F35-GFP, $8 \%$ and $7 \%$ respectively (Fig. 4). In contrast, only 


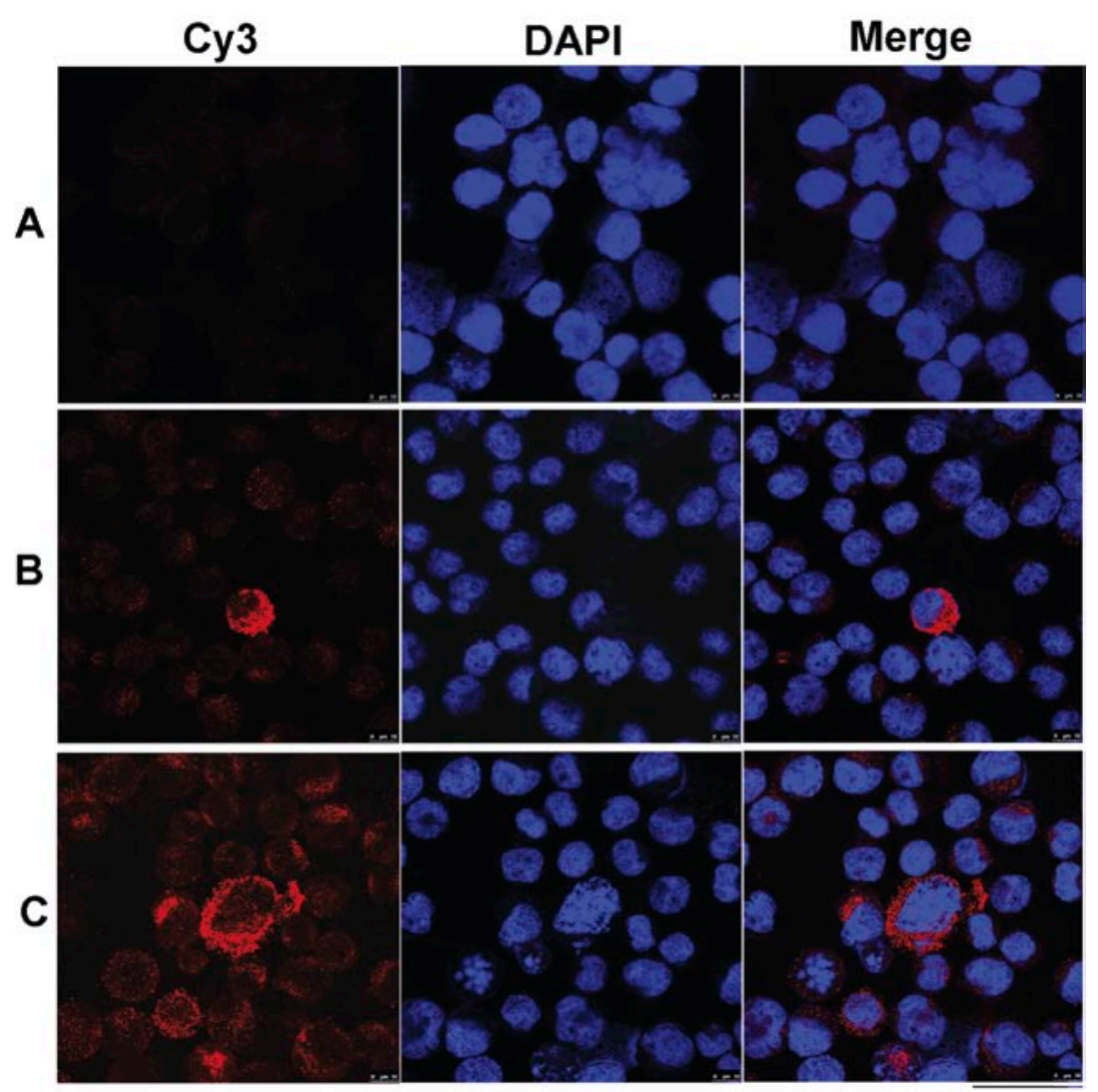

Figure 2. Cytoplasmic localization of the exogenous SH2-HA fusion protein after its infection into K562 cells as observed under the confocal microscopy. (A) Ad5/F35-GFP infected K562 cells; (B) Ad-SH2-HA infected K562 cells; (C) Ad-Sm-HA infected K562 cells. K562 cells, treated respectively with Ad5/ F35-GFP, Ad-SH2-HA or Ad-Sm-HA, were stained with anti-HA antibody and Cy3-labeled goat anti-mouse IgG, followed by DAPI conterstaining. Four consecutive confocal images were merged for each picture.
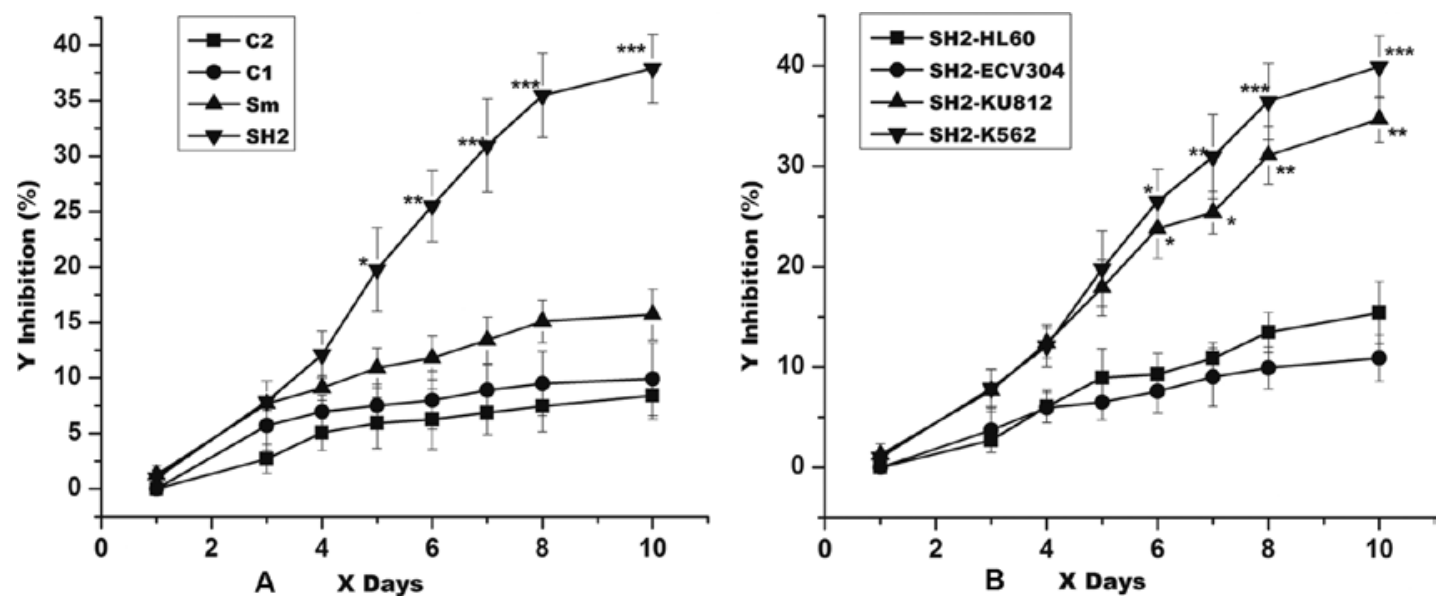

Figure 3. Effects of Ad-SH2-HA infection on the cell growth by MTT assay. (A) K562 cells were treated with Ad-SH2-HA, Ad-Sm-HA or Ad5/F35-GFP or simply treated with an equal volume of PBS for 1-10 days, and cell growth was determined by MTT assay. Data are representative of three independent measurements and the standard errors of the means are shown. (B) K562, KU812, HL-60 and ECV304 cells were treated with Ad-SH2-HA for 1-10 days, and cell growth was determined by MTT assay. Data are representative of three independent measurements and the standard errors of the means are shown. ${ }^{*} \mathrm{p}<0.05,{ }^{* *} \mathrm{p}<0.01,{ }^{* * *} \mathrm{p}<0.001$ vs. control groups.

a modest fraction of Ad-Sm-HA -infected K562 and KU812 cells underwent apoptosis (12\% for K562 and 13\% for KU812) (Fig. 4).

Ad-SH2-HA infection functions by binding to the phosphoBcr-Abl Y177 site, competitively disrupting the Grb2
SH2-phospho-Bcr-AblY177complex.Co-immunoprecipitation and immunoblotting were employed to confirm the disrupting effect of Ad-SH2-HA infection on the interaction between the endogenous $\mathrm{SH} 2$ and the BCR-ABL oncoprotein. Human K562 cells were infected with the recombinant Ad-SH2-HA or Ad-Sm-HA adenovirus at a final MOI of 10000. Cells 


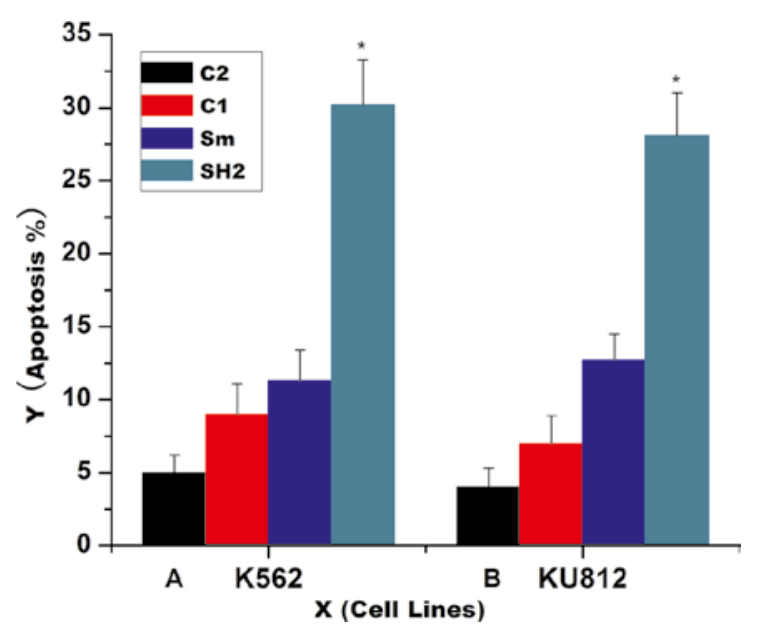

Figure 4. Effects of Ad-SH2-HA infection on the induction of cell apoptosis as analyzed by flow cytometry. K562 (A) and KU812 (B) cells were respectively infected with Ad-SH2-HA, Ad-Sm-HA or Ad5/F35-GFP or simply treated with an equal volume of PBS for 5 days, and cells were stained with Annexin V-PE and PI and analyzed by flow cytometry. "p $<0.05$ vs. control groups.

were lyzed, and anti-HA agarose slurry $(6 \mu \mathrm{l})$ was incubated with $500 \mu 1$ cell lysate from Ad-SH2-HA (Fig. 5A, Lane 1), Ad-Sm-HA infected (Fig. 5A, Lane 2), Ad5/F35-GFP infected (Fig. 5A, Lane 3) or PBS treated cells (Fig. 5A, Lane 4). IP and Co-IP reactions were performed at $4^{\circ} \mathrm{C}$ overnight. IP and Co-IP products were eluted with $25 \mu \mathrm{l}$ non-reducing sample buffer. The upper half of the Western blot membrane was probed with anti-phospho-Bcr-Abl Y177 antibody and the lower half with anti-HA antibody. HA-tagged SH2-HA were immunoprecipitated by anti-HA antibody (Fig. 5B, lower Lane 1), and BCR-ABL oncoprotein was co-immunoprecipitated with SH2-HA (Fig. 5A, lower Lane 1). Reciprocally, Bcr-Abl oncoprotein was immunoprecipitated by the anti-phospho-BcrAbl Y177 antibody (Fig. 5B, Lane 1), and HA-tagged SH2-HA was co-immunoprecipitated with anti-phospho-Bcr-Abl Y177 (Fig. 5B, Lane 1).

Ad-SH2-HA infection reduced Ras, MAPK and Akt Kinase activities in $\mathrm{K5} 62$ cells. We examined the potential effect of Ad-SH2-HA infection on the reduction of Ras, MAPK and Akt kinase activities downstream of the direct interaction between the Grb2 SH2 domain and the phosphorylated Bcr-Abl Y177 motif. BCR/ABL-Y177 interaction with Grb2 potentially promotes association of Grb2-SH3 domains with the guanine nucleotide exchange factor SoS, which can activate Ras by stimulating exchange of GDP for GTP (24). We expectedly observed significantly decreased levels of active GTP-bound Ras and reduced Ras activity in Ad-SH2-HA infected, but not the Ad-Sm-HA infected, K562 cells (Fig. 6A). These results indicate a critical effect of the exogenous SH2-mediated disruption of Grb2 SH2-phospho-Bcr-Abl Y177 interaction in Ad-SH2-HA infection-induced inactivation of Ras in CML cells. MAPK is activated downstream of Ras (25). Western blotting with phospho-MAPK antibodies revealed that P-MAPK levels were concordantly reduced in Ad-SH2-HA infected K562 cells (Fig. 6B), but not in Ad-Sm-HA infected cells compared with the control adenovirus-treated cells. These results were further confirmed by direct assessment of MAPK activity using a substrate phosphorylation assay (Fig. 6B). Grb2-Gab2-PI3K-Akt pathway downstream of Grb2 SH2-phospho-Bcr-Abl Y177 interaction may contribute to protection from enhanced proliferation and survival of BCR/ ABL-expressing cells. PI3K consists of a regulatory $85-\mathrm{kDa}$ subunit and a catalytic $110-\mathrm{kDa}$ subunit (26). Akt is a key downstream effector of PI3K signaling. Western blotting with anti-phospho-Akt $\left(\mathrm{Ser}^{473}\right)$ antibodies showed decreased Akt phosphorylation, indicating reduced activation of Akt kinase activity in Ad-SH2-HA infected K562 cells, which could not be observed in the Ad-Sm-HA infected K562 cells (Fig. 6C). These results were further confirmed by direct assessment of Akt activity in a substrate-phosphorylation assay (Fig. 6C).

\section{Discussion}

In the present study, we have examined the ability of the exogenous $\mathrm{SH} 2$ domain of the Grb2 protein to localize into the cytoplasmic compartment, to heterodimerize with the phospho-Bcr-Abl Y177 fusion protein, to inhibit the growth of the CML cells, and to disrupt the Grb2-SoS-Ras-MAPK and Grb2-Gab2-PI3K-Akt pathways downstream of the Bcr-Abl oncoprotein. Efficient infection of the Ad-SH2-HA adenovirus and the potent expression of the SH2-HA fusion protein in CML cells were confirmed respectively by immunofluorescence microscopy and Western blotting. Furthermore, as demonstrated by confocal microscopy, the recombinant
SH2 Sm C1 C2

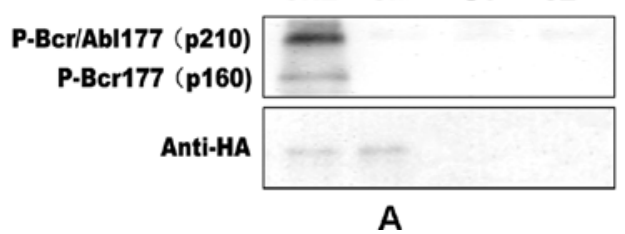

SH2 Sm C1 C2

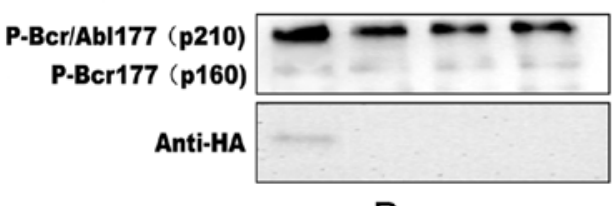

B

Figure 5. Co-IP of active HA-tagged SH2-HA with phospho-Bcr-Abl Y177. Panels are respectively the upper and the lower half of the same Western blot respectively probed with anti-phospho-Bcr-Abl Y177 antibody and anti-HA antibody. Human K562 cells were infected with Ad-SH2-HA, Ad-Sm-HA or Ad5/F35-GFP or simply treated with an equal volume of PBS for $48 \mathrm{~h}$. Immunoprecipitations of the cell lysates were carried out with anti-HA (A) or antiphospho-Bcr-Abl Y177 antibodies (B). Immunoprecipitates were subjected to immunoanalysis with anti-phospho-Bcr-Abl Y177 (A) or anti-HA antibodies (B). SH2, Ad-SH2-HA infected K562 cell lysate; Sm, Ad-Sm-HA infected K562 cell lysate; C1, Ad5/F35-GFP infected K562 cell lysate; C2, PBS treated K562 cell lysate. Data shown are the representative of three independent experiments with similar results. 


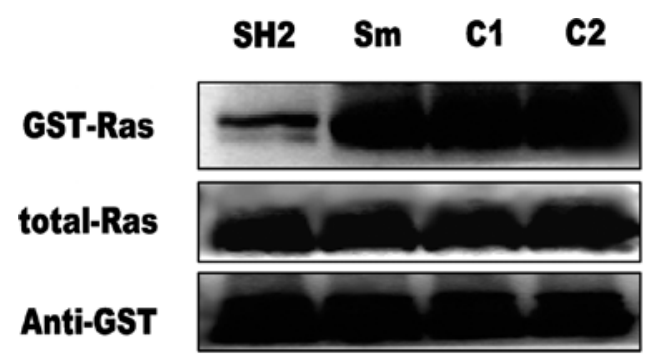

A

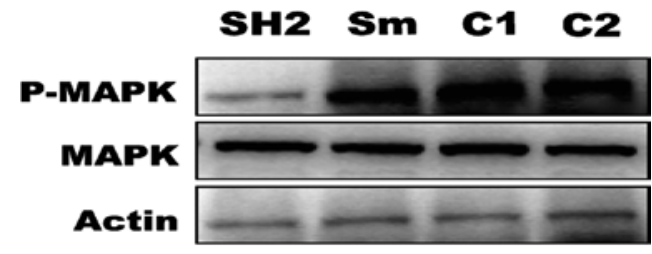

MAPK kinase assay

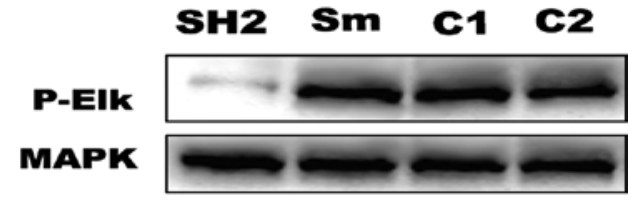

B

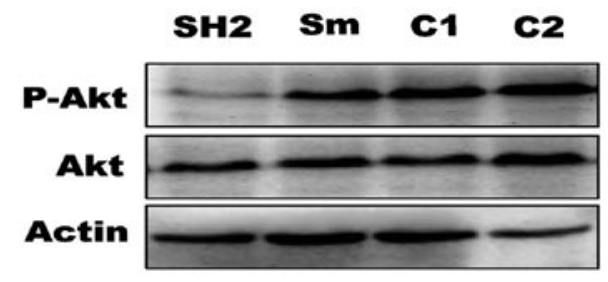

Akt kinase assay

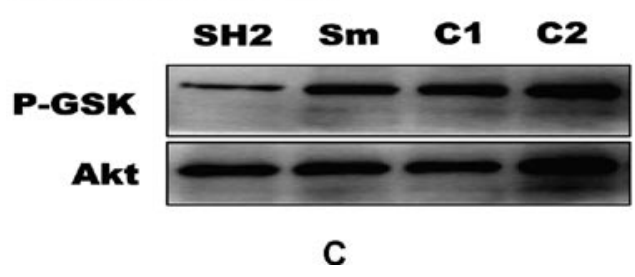

Figure 6. Effect of Ad-SH2-HA infection on Ras, MAPK and Akt kinase activities in K562 cells. Protein extracts from K562 cells treated with the Ad-SH2-HA (SH2), Ad-Sm-HA (Sm), Ad5F35-GFP (C1) or PBS (C2) were analyzed. (A) Ras, Ras activity assays were done using GST-Raf-1-RBD agarose-conjugated beads to bind Ras-GTP, which was detected by Western blotting with an anti-Ras antibody, and compared with total Ras. Blots were reprobed with an anti-GST antibody to assess loading of GST-fusion protein. (B) MAPK, Western blot analysis of phospho-MAPK and total MAPK levels in adenovirus-infected cells was done. Blots were reprobed for $\beta$-actin to assess protein loading. MAPK kinase activity assays were done using MAPK immunoprecipitated from infected cells and in vitro kinase assays using an ERK-1 substrate followed by Western blotting with an antiphospho-ERK-1 antibody. A fraction of the lysates used for these assays was reserved for Western blotting to detect input levels of MAPK in the samples. (C) Akt, Western blot analysis of phosphorylated (Ser473) Akt and total Akt levels in infected cells was done. Akt kinase assays were done using Akt immunoprecipitated from transduced cells and in vitro kinase assays using a GSK-3 substrate followed by Western blotting with an anti-phospho-GSK-3 antibody. A fraction of the lysates used for these assays was reserved for Western blotting to detect input levels of Akt in the samples.
$\mathrm{SH} 2-\mathrm{HA}$ protein was found out to be located in the cytoplasm of K562 cells, where most of the Bcr-Abl oncoprotein resides. Moreover, we demonstrated the adenovirally expressed exogenous $\mathrm{SH} 2$ peptides not only strongly inhibited the proliferation of the CML cells, but also significantly induced their apoptosis. Besides the expected heterodimerization of the exogenous SH2-HA peptide with the phospho-Bcr-Abl Y177 domain of the Bcr-Abl oncoprotein, it was observed that SH2-HA protein potently inhibited the kinase activities of Ras, MAPK and PI3K downstream of the Bcr-Abl oncoprotein.

Grb2-SH3 domain blocking peptides have already shown biologic activity in CML cells. Several of these studies have used cell-penetrating shuttle peptides to transport the $\mathrm{SH} 3$ domain-specific blocker peptides into CML cells. For example, Ye et al (27) have used a Grb2-SH3 inhibitor called peptidimer-c, which was demonstrated to disrupt the Grb2-SoS complex and showed cell cycle arrest effect on the Bcr-Abl positive K562 cells; Kardinal et al (28) have tested another Grb2-SH3 domain blocker named HAGBPs, which was shown to have successfully induced a dramatic decrease in the proliferation of both CML cell lines and the freshly isolated CML blast cells from patients, with expected down-regulation of Grb2-SoS complexes and MAPK activity. However, the long-term potential of peptide- or peptoid-based therapeutics that target intracellular signal-transduction components is still largely unclear. There is still a long way to go before methods can be found to help to develop the peptides currently at hand into clinically valuable molecules.

Compared with the conventional Grb2-SH3 domain blocking peptides, the present Ad-SH2-HA adenovirus-mediated $\mathrm{SH} 2$ domain blocking strategy is expected to be much more efficacious. Indeed, since numerous signaling pathways including both the Grb2-SoS-Ras-MAPK and Grb2-Gab2-PI3K-Akt pathways are activated upon the Grb2-Bcr-Abl interaction in CML cells $(7,8)$, the Grb2-Bcr-Abl complex-disrupting strategy through large excess of exogenous $\mathrm{SH} 2$ is expected to show an epistatic effect on the inhibition of oncogenic signaling pathways downstream of Bcr-Abl. On the other hand, by showing that the Ad5F35 vector-mediated gene delivery into CML cells can mount an effective therapeutic response against $\mathrm{CML}$ cells by delivery of genes capable of inhibiting the oncogenic signaling in CML, our present study has further confirmed that the new Ad5F35 vectors designed by Nilsson et al (15) could facilitate studies of hematopoietic malignancies when transient gene delivery is desirable. Moreover, we are confident that purging of malignant hematopoietic cells can be explored by the Ad5F35 vector-mediated delivery of pro-apoptotic genes or suicide genes in a tumor cell specific manner.

In comparison with the traditional small molecular kinase inhibitors, the present design is bound to be much more promising. Since all small tyrosine kinase inhibitor resistances are clearly due to mutations in the tyrosine kinase active site of Bcr-Abl and since exogenous $\mathrm{SH} 2$ acts downstream the protein, its effect on small tyrosine kinase inhibitor-resistant clones might be similar to that on small tyrosine kinase inhibitor-sensitive ones. Experiments to explore the possible anti-CML efficacy of Ad-SH2-HA in murine BaF3-p210 cells harbouring different Bcr-Abl mutational states comprising the imatinib-resistant mutants M351T, E255K and T315I are currently underway. 
In summary, we have constructed the adenovirus for the SH2 domain of the Grb2 protein, and the Ad-SH2-HA adenovirus showed potent infection efficiency in CML cells. Moreover, we demonstrated the proliferation-inhibiting and apoptosis-inducing effect of the adenovirally expressed exogenous SH2 peptides on CML cells. Furthermore, by showing that the Grb2-SoS-Ras-MAPK and Grb2-Gab2PI3K-Akt pathways downstream of the Bcr-Abl oncoprotein were potently inhibited after the exogenous SH2-HA fusion protein is localized into the cytoplasmic compartment and heterodimerized with the phospho-Bcr-Abl Y177 oncoprotein, we could demonstrate the oncogenecity inhibitory effect of the adenovirally expressed SH2-HA fusion protein on the Bcr-Abl oncoprotein. The legitimacy of the SH2-HA fusion protein as a phospho-Bcr-Abl Y177 kinase inhibitor was proven by abolishing the heterodimerization and kinase inhibiting activity through the treatment of the mutant Ad-Sm-HA adenovirus, in which two base pairs of the $\mathrm{SH} 2$ gene were mutated, leading to one amino acid mutation (R27K) in the binding domain, which was expected to hinder its binding to phospho-Bcr-Abl Y177. Experiments to probe into the possible role the recombinant SH2-HA fusion protein plays in triggering CML cell apoptosis both in vitro and in vivo are underway. Creating an adenovirus encoding the deletion mutants of the $\mathrm{SH} 2$ domain of the Bcr-Abl Grb2 protein, and proteomic studies to identify the downstream interacting partners of SH2 will further our understanding on the role of this domain in the physiology and pathology of the CML disease. Other intriguing features of SH2-HA, including its specific toxicity in Bcr-Abl positive leukemia cells and specific organ tropisms, remain to be further explored, and it is hoped that the Ad-SH2-HA adenovirus will eventually show potential value for a range of therapeutic applications such as purging of autologous bone marrow as used for the treatment of CML and may possibly direct treatment of leukemias either alone or combined together with other targeted molecules for targeting of Bcr-Abl positive leukemias in the future.

\section{Acknowledgments}

We thank Xiaolong Fan (the Lund University, Sweden) and the members of his group at Lund University for Ad5/f35GFP vectors. This study was supported by the National Science Foundation of China Grant No. 30871102 to W.L.F. and the Doctor Foundation of Chongqing Medical University 2009 to Z.P.

\section{References}

1. Quintás-Cardama A and Cortes J: Molecular biology of bcr-abl1positive chronic myeloid leukemia. Blood 113: 1619-1630, 2009.

2. Baccarani M, Cortes J, Pane F, et al: Chronic myeloid leukemia: an update of concepts and management recommendations of European Leukemia Net. J Clin Oncol 27: 6041-6051, 2009.

3. Gorre ME, Mohammed M, Ellwood K, Hsu N, Paquette R, Rao PN and Sawyers CL: Clinical resistance to STI-571 cancer therapy caused by BCR-ABL gene mutation or amplification. Science 293: 876-880, 2001.

4. Kantarjian HM, Giles F, Quintás-Cardama A and Cortes J: Important therapeutic targets in chronic myelogenous leukemia. Clin Cancer Res 13: 1089-1097, 2007.
5. Quintás-Cardama A and Cortes J: Therapeutic options against BCR-ABL1 T315I-positive chronic myelogenous leukemia. Clin Cancer Res 14: 4392-4399, 2008.

6. Million RP and van Etten RA: The Grb2 binding site is required for the induction of chronic myeloid leukemia-like disease in mice by the Bcr/Abl tyrosine kinase. Blood 96: 664-670, 2000.

7. Ren R: Mechanisms of BCR-ABL in the pathogenesis of chronic myelogenous leukaemia. Nat Rev Cancer 5: 172-183, 2005.

8. Chu S, Li L, Singh H and Bhatia R: BCR-tyrosine 177 plays an essential role in Ras and Akt activation and in human hematopoietic progenitor transformation in chronic myelogenous leukemia. Cancer Res 67: 7045-7053, 2007.

9. Check E: Gene therapy: shining hopes dented - but not dashed. Nature 420: 735, 2002.

10. Connolly JB: Lentiviruses in gene therapy clinical research. Gene Ther 9: 1730-1734, 2002.

11. Cornetta K, Morgan RA and Anderson WF: Safety issues related to retroviral-mediated gene transfer in humans. Hum Gene Ther 2: 5-14, 1991

12. Marshall E: Viral threat to newborns under radar. Science 295: 1631,2002

13. Bajocchi G, Feldman SH, Crystal RG and Mastrangeli A: Direct in vivo gene transfer to ependymal cells in the central nervous system using recombinant adenovirus vectors. Nat Genet 3: 229-234, 1993.

14. Neering SJ, Hardy SF, Minamoto D, Spratt SK and Jordan CT: Transduction of primitive human hematopoietic cells with recombinant adenovirus vectors. Blood 88: 1147-1155, 1996.

15. Nilsson M, Ljungberg J, Richter J, et al: Development of an adenoviral vector system with adenovirus serotype 35 tropism; efficient transient gene transfer into primary malignant hematopoietic cells. J Gene Med 6: 631-641, 2004.

16. Pendergast AM, Quilliam LA, Cripe LD, et al: BCR-ABLinduced oncogenesis is mediated by direct interaction with the SH2 domain of the GRB-2 adaptor protein. Cell 75: 175-185, 1993.

17. Luo J, Deng ZL, Luo X, et al: A protocol for rapid generation of recombinant adenoviruses using the AdEasy system. Nat Protoc 2: 1236-1247, 2007.

18. Fan X, Brun A and Karlsson S: Adenoviral vector design forhigh level transgene expression in primitive human hemtopoietic progenitors. Gene Ther 7: 2132-2138, 2000.

19. Peng Z, Xiao Z, Wang Y, et al: Reversal of P-glycoproteinmediated multidrug resistance with small interference RNA (siRNA) in leukemia cells. Cancer Gene Ther 11: 707-712, 2004.

20. Huang SF, Liu DB, Zeng JM, et al: Cloning, expression, purification, distribution and kinetics characterization of the bacterial beta-galactosidase fused to the cytoplasmic transduction peptide in vitro and in vivo. Protein Expr Purif 68: 167-176, 2009.

21. Huang SF, Liu DB, Zeng JM, et al: Cloning, expression, purification and functional characterization of the oligomerization domain of Bcr-Abl oncoprotein fused to the cytoplasmic transduction peptide. Protein Expr Purif 64: 167-178, 2009.

22. Chu S, Holtz M, Gupta M and Bhatia R: BCR/ABL kinase inhibition by imatinib mesylate enhances MAP kinase activity in chronic myelogenous leukemia CD $34^{+}$cells. Blood 103: 3167-3174, 2004.

23. Chu S, Li L, Singh H and Bhatia R: BCR-tyrosine 177 plays an essential role in Ras and Akt activation and in human hematopoietic progenitor transformation in chronic myelogenous leukemia. Cancer Res 67: 7045-7053, 2007.

24. Gishizky ML, Cortez D and Pendergast AM: Mutant forms of growth factor-binding protein-2 reverse BCR-ABL-induced transformation. Proc Natl Acad Sci USA 92: 10889-10893, 1995.

25. Kolch W: Coordinating ERK/MAPK signalling through scaffolds and inhibitors. Nat Rev Mol Cell Biol 6: 827-837, 2005.

26. Shaw RJ and Cantley LC: Ras, PI3K and mTOR signalling controls tumour cell growth. Nature 441: 424-430, 2006.

27. Ye YB, Lin JY, Chen Q, et al: The cytotoxicity of a Grb2-SH3 inhibitor in Bcr-Abl positive K562 cells. Biochem Pharmacol 75: 2080-2091, 2008.

28. Kardinal C, Konkol B, Lin H, et al: Chronic myelogenous leukemia blast cell proliferation is inhibited by peptides that disrupt Grb2-SoS complexes. Blood 98: 1773-1781, 2001. 